\title{
Exploiter le potentiel des processus de renormalisation en formation à l'enseignement
}

Using The Potential of Renormalization Processes In Teacher Education

\section{Valérie Lussi Borer et Alain Muller}

\section{(2) OpenEdition}

\section{Journals}

Édition électronique

URL : http://journals.openedition.org/activites/967

DOI : 10.4000 /activites.967

ISSN : $1765-2723$

Éditeur

ARPACT - Association Recherches et Pratiques sur les ACTivités

Référence électronique

Valérie Lussi Borer et Alain Muller, «Exploiter le potentiel des processus de renormalisation en formation à l'enseignement », Activités [En ligne], 11-2 | Octobre 2014, mis en ligne le 15 octobre 2014 consulté le 01 mai 2019. URL : http://journals.openedition.org/activites/967 ; DOI : 10.4000/ activites.967

\section{(c) (i) (9)}

Activités est mis à disposition selon les termes de la licence Creative Commons Attribution - Pas d'Utilisation Commerciale - Pas de Modification 4.0 International. 


\title{
Exploiter le potentiel des processus de renormalisation en formation à l'enseignement
}

\author{
Valérie Lussi Borer
}

\author{
Laboratoire CRAFT \\ Institut Universitaire de Formation des Enseignants \& Faculté de Psychologie et des Sciences de l'éducation, \\ Université de Genève, Bd. du Pont-d'Arve 40,1205 Genève \\ Valerie.Lussi@unige.ch \\ Alain Muller \\ Laboratoire CRAFT \\ Institut Universitaire de Formation des Enseignants \& Faculté de Psychologie et des Sciences de l'éducation, \\ Université de Genève, Bd. du Pont-d'Arve 40,1205 Genève
}

Alain.Muller@unige.ch

\begin{abstract}
Using The Potential Of Renormalization Processes In Teacher Education. The article analyzes the potential of renormalization contained in "activity oriented" training devices using the viewing of films showing teachers at work. We define renormalization as the permanent work of renewal 1) of the rules used to develop the experience and 2) of the rules to be followed in the action. The analysis focuses 1) on an "Other-Confrontation Interview" sequence (film confronting a teacher with the work of another teacher that he/she does not know), 2) on a "Self-Confrontation Interview" sequence (film confronting a teacher with his/her own work) followed by a "Cross-Confrontation Interview" (film confronting two teachers with the work of one of them). These sequences come from research conducted with secondary teachers trained at the University of Geneva. The processes of renormalization are analysed. Step by step, the analyses follow teachers' interactions with the films viewed. They show that these processes of renormalization hang upon a double movement of the clarification of implicit rules, and the development of conflicts between the personal rules and the rules of the environment (institution and expectations of peers). We discuss the potential of these processes for teacher education and propose a curriculum to promote them.
\end{abstract}

KEYWORDS

activity, teacher education, renormalization, video training

\section{Introduction}

Cet article s'inscrit dans le cadre de recherches portant sur les processus de formation suscités par le visionnement de films montrant des enseignants au travail (Lussi Borer \& Muller, 2014, à paraître a, b). Ces recherches, menées avec des enseignants secondaires en formation (EEF) ont révélé, entre autres, que le visionnement de films et les commentaires que ce visionnement génère chez les enseignants en formation peuvent déclencher des processus de renormalisation, soit de transformation des règles à suivre dans l'action. Dans le but d'investiguer plus finement ces processus, nous soumettons à l'analyse 1) une séquence d'alloconfrontation, 2) une séquence d'autoconfrontation simple suivie d'une séquence d'autoconfrontation croisée. Ces analyses s'ouvrent sur une discussion concernant 
l'usage qu'il peut être fait de ces processus dans le cadre d'une ingénierie de formation visant à favoriser leur émergence.

\section{1.- Présentation de la recherche}

\section{1.- Ancrage conceptuel général}

En premier lieu, et de manière générale, notre recherche s'inscrit dans le courant d' « analyse de l'activité ». De manière plus spécifique, elle s'appuie sur une perspective développementale prenant, entre autres, sa source dans le pragmatisme de Dewey (1990), perspective selon laquelle les objectifs de formation doivent viser en premier lieu à libérer l'activité et non pas à la contraindre. Ils doivent ainsi être "dégagés » de l'intérieur de l'activité - c'est-à-dire exprimer ce qui « est en germe » dans celle-ci - et non pas s'imposer à elle de l'extérieur, bref doivent permettre d'en réaliser les potentialités présentes.

Le développement des personnes en formation consiste en la transformation de leurs dispositions à agir, que nous concevons, à la suite de Lahire (1998) et Leblanc (à paraître) comme des propensions ou des tendances des personnes à agir d'une certaine manière dans des circonstances déterminées. Cette transformation des dispositions à agir procède, entre autres, de processus de renormalisation (Schwartz, 2007; Schwartz \& Durrive, 2009) soit d'un travail permanent de renouvellement des règles d'élaboration de l'expérience et des règles à suivre dans l'action.

Sur ce soubassement conceptuel, l'hypothèse qui soutient notre recherche est que, soit en relation directe à leur activité dans les cas d'autoconfrontation, soit en relation indirecte à leur activité, c'est-à-dire par expérience mimétique (Durand, 2008 ; Zaccaï-Reyners, 2005) dans les cas d'alloconfrontation, le double travail de visionnement et de commentaire d'une activité d'enseignement permet une certaine transformation des dispositions à agir des personnes en formation en ce qu'il suscite des processus de renormalisation.

\section{2.- Règles, renormalisation, dispositions à agir}

«C'est un caractère élémentaire des croyances comme des actions, parce qu'elles prennent leur sens dans un contexte public, que de jouer un rôle régulateur, lié aux évaluations qu'elles autorisent ou aux fins qui leur sont associées, et par conséquent de se conjuguer à des règles » (Cometti, 2011, pp. 13-14). Toute action est ainsi en soi régulatrice et réglée: règles et actions sont dans une relation interne (logique) et non externe (causale, la règle déterminant de l'extérieur l'action). «La présence d'une obligation ressentie comme telle, soit encore [...] la distinction qu'on est en mesure ou non d'établir entre une manière correcte et une manière incorrecte de faire» (Cometti, 2011, p. 15) témoigne d'une normativité opérante.

On peut distinguer les règles, d'une part selon qu'elles sont exogènes ou endogènes (Venner \& Schwartz, 2009), d'autre part selon qu'elles sont explicites ou implicites (Cometti, 2011 ; Winch, 2009). Les règles exogènes émises dans le milieu de travail peuvent être explicitement formalisées dans des textes, comme par exemple des plans d'études, des moyens d'enseignement, un code de déontologie. Mais le milieu « sécrète » aussi des normes qui opèrent de manière beaucoup plus implicite. Il en est ainsi des attentes des pairs fonctionnant comme des allants de soi, au sein d'une certaine culture de métier informelle, ou de ce que les cliniciens de l'activité appellent le genre professionnel: «le genre est en quelque sorte la partie sous-entendue de l'activité, ce que les travailleurs d'un milieu donné connaissent et voient, attendent et reconnaissent, apprécient ou redoutent; ce qui leur est commun et qui les réunit sous des conditions réelles de vie; ce qu'ils savent devoir faire grâce à une communauté d'évaluations présupposées, sans qu'il soit nécessaire de respécifier la tâche chaque fois qu'elle se présente » (Clot \& Faïta, 2000, p. 11). 
Les règles endogènes sont celles appartenant aux individus. Du côté des règles endogènes implicites, nous versons les dispositions à agir. Nous entendons par disposition à agir, une habitude au sens de Dewey, soit « une organisation dynamique de l'activité et non une entité interne improbable ou un mécanisme causal» (Garetta, 2002, p. 140). La disposition à agir, n'est pas détachée de l'activité et ne précède pas celle-ci, elle est «l'activité qui s'organise elle-même : redéfinitions de situations, habitudes «cadrant» la singularité de l'activité en cours, anticipations de ce qui pourrait arriver, etc., les dispositions sont bien le «pôle » organisateur ou structurant de l'activité » (Muller, 2014, p. 192). Mais les règles personnelles organisant l'activité peuvent aussi être explicites. Elles peuvent consister en des connaissances ou croyances communicables, acquises de manière formelle ou informelle. Elles peuvent aussi trouver leur origine dans l'explicitation langagière des règles implicites, dans un travail de «prise de conscience » qui advient souvent quand les individus sont tenus de commenter leur action, ou, quand, devant l'échec de celle-ci, ils sont poussés à la prendre comme objet de réflexion.

Nous empruntons la notion de renormalisation à Schwartz (Schwartz, 2007 ; Schwartz \& Durrive, 2009). Selon lui le processus de renormalisation consiste en une ré-interprétation des normes émises par le milieu, mais aussi des normes endogènes, en vue de configurer ce milieu comme étant le sien. Les normes sont ainsi transformées «sous le coup» de la singularité des situations. Mais il nous semble possible - c'est du moins dans ce sens que nous l'entendrons dans ce texte - d'étendre la notion à l'ensemble du travail permanent que les différentes instances normatives opèrent les unes sur les autres: interprétation des prescriptions à l'aune des normes individuelles (implicites ou explicites); médiation opérée par le genre entre prescriptions et activité individuelle ; interprétation du genre par le style personnel, mais aussi transformation du genre sous le coup des évolutions des styles; altération des dispositions à agir par «prise de conscience » ou incorporation d'un genre; et encore, transformation des prescriptions sous la pression d'une activité réelle qui arrive à se faire reconnaître; etc. Bref, endogène, exogène, implicite et explicite, ne sont pas des «mondes» séparés, mais des instances organisatrices de l'action, qui, par leur interpénétration permanente, ne cessent de se (re)produire tout en s'altérant. Ou plutôt, devrions-nous dire, ne devraient cesser de se (re)produire tout en s'altérant. Car dans certaines situations «pathologiques» de travail ou de formation, ces instances n'interagissent plus entre elles, chacune se rétractant en elle-même, s'opposant aux autres sans les féconder, ce qui signe le blocage du processus de développement des personnes.

\section{3.- Contexte de la recherche}

Nos recherches, initiées début 2011, sont menées avec des enseignants secondaires en formation. Ceux-ci suivent deux années de formation à l'Institut Universitaire de Formation des Enseignants de Genève. Certains d'entre eux sont déjà en emploi, d'autres pas encore. Les EEF ayant participé à ces recherches - une trentaine jusqu'à présent - ont soit travaillé sur des films de la plateforme Néopass@ction (alloconfrontation), soit travaillé sur leur propre activité filmée (autoconfrontation).

Les enseignants en deuxième année de formation et en emploi - c'est le cas des trois enseignantes dont l'activité fait l'objet des analyses qui suivent - sont soumis à une situation complexe et souvent vécue avec difficulté, relative à leur double « allégeance » à l'institut de formation et à un «terrain » qu'ils ne connaissent pas bien. Ils sont tenus de répondre à un faisceau de prescriptions (de normes exogènes) multiples, souvent contradictoires et peu opérationnelles à leur stade de développement professionnel.

Premièrement, les normes de l'institut de formation et celles du terrain sont bien souvent en opposition. Deuxièmement, il n'y pas forcément de cohérence non plus dans les normes émises à l'intérieur de chacune des deux instances. Troisièmement, les EEF vivent les prescriptions de l'institut de formation comme en même temps très contraignantes - ils sont 
tenus de s'y soumettre en vue de l'évaluation certificative de leurs compétences - et trop génériques pour qu'ils puissent en faire quelque chose dans leur pratique de tous les jours (Méard \& Bruno, 2009). Ainsi, les EEF ont l'impression qu'on leur prescrit fortement ce qu'il faut faire, mais sans leur dire comment le faire. À cela s'ajoute le poids des prescriptions qu'ils se donnent à eux-mêmes (normes endogènes) et qui, eu égard à leur position de débutant dans le métier, ne sont pas toujours très « réalistes ». En résumé, les EEF sont non seulement confrontés à la nécessité que rencontre tout travailleur de faire siennes les normes émises par le milieu, mais plus encore, de « désopacifier », tant en ce qui concerne leur contenu que leur origine, le tissu serré et contradictoire de celles-ci : bien avant de pouvoir renormaliser il leur faut d'abord comprendre ce que sont les normes et d'où elles viennent.

\section{4.- Procédure de recueil de données et choix des extraits analysés}

Les données présentées dans cette recherche ont été produites en recourant à trois procédures de recueil : alloconfrontation, autoconfrontation simple, autoconfrontation croisée.

Ce travail a été mené dans la perspective d'une enquête inter-objective (Zask, 2004), pour laquelle un chercheur-formateur $(\mathrm{CF})$ vise à co-construire avec les EEF concernés un objet d'enquête partagé : les interlocuteurs, chacun du point de vue qui est le leur et de la position qu'ils occupent, font émerger pas à pas, et à travers leur dialogue, des « objets de préoccupation commune ». ${ }^{1}$ La personne menant l'entretien n'est donc ni «du pur côté » et au service de la recherche, ni «du pur côté » et au service du développement de l'autre : il est chercheur-formateur en étant avec l'autre dans l'élaboration d'un objet de recherchedéveloppement commun.

Les entretiens n'ont donc pas été menés dans une unique perspective de recueil de données complémentaires à celles de l'observation permettant de documenter l'activité en vue d'une modélisation de celle-ci, comme c'est le cas dans la perspective du «cours d'action» (Theureau, 2010). Nous n'avons pas œuvré non plus, même si nous en sommes proches, dans la perspective de la clinique de l'activité pour laquelle il s'agit soit, dans le cas de l'autoconfrontation simple, de susciter une ressaisie langagière de l'activité autorisant une restructuration de celle-ci en vue de son déploiement ultérieur, soit, dans le cas de l'autoconfrontation croisée de susciter des controverses «nourrissant» le genre professionnel (Clot, Faïta, Fernandez \& Scheller, 2000).

Notre perspective d'enquête inter-objective a deux conséquences principales sur le déroulement des entretiens. Premièrement, dans la mesure où il s'agit de construire un objet commun, le chercheur-formateur peut être conduit à intervenir assez souvent et par moments de manière non neutre en proposant son point de vue. Deuxièmement, eu égard encore une fois à cette co-construction d'objet d'enquête, l'entretien tend assez régulièrement à « décrocher » de la pure documentation de l'activité, pour « dériver» vers des commentaires plus généraux : moments d'analyse, comparaisons d'activités, esquisses de transformations possibles de l'activité, etc.

Plus précisément, dans les cas d'alloconfrontation, il a été demandé aux enseignants en formation (EEF) de naviguer sur la plateforme Néopass@ction et de commenter les «contours» de leur activité, soit ce que suscite chez eux ce qu'ils voient, en étant accompagnés par un CF. Le rôle du CF était tout aussi peu contraint : questions, demandes de précisions, entrée dans de courts dialogues, etc. Était visé en premier lieu le recueil de

\footnotetext{
Cette co-construction peut se faire à deux niveaux. L'objet même d'enquête - ce à quoi on va s'intéresser peut émerger en cours d'entretien, sans avoir été défini préalablement à celui-ci. C'est ce qui s'est passé dans notre recherche avec les entretiens d'alloconfrontation. Mais la co-construction peut aussi concerner le « contenu » d'un objet d'enquête pré-défini en amont de l'entretien. Ce qui émerge dans le dialogue concerne dans ce cas ce qu'il en est de l'objet auquel on s'intéresse. C'est ce qui s'est passé dans notre recherche avec les entretiens d'autoconfrontation simple et croisée.
} 
l'empan le plus large de réactions aux activités visionnées, la «mise en lumière » de tout ce qu'il est possible de dire de ces activités. Ces séances d'une durée de \pm 60 minutes ont été filmées, puis retranscrites sous forme de verbatim.

Dans les cas d'autoconfrontation, les EEF ont défini en groupe (de deux ou plus) une thématique d'enquête issue de leurs préoccupations actuelles qu'ils ont contractualisées avec le CF. Ils ont ensuite filmé une séquence de classe (environ $45 \mathrm{mn}$ ) et ont sélectionné un extrait d'environ $15 \mathrm{mn}$ dans lequel cette thématique est présente. Ils ont ensuite travaillé avec le CF en autoconfrontation sur l'extrait sélectionné (env. $60 \mathrm{mn}$ ) puis en autoconfrontation croisée ou collective avec les autres EEF sur le même extrait (env. $120 \mathrm{mn})$.

Les trois extraits analysés ont été choisis parmi l'ensemble du corpus recueilli, non pas parce qu'ils sont représentatifs de celui-ci, mais parce qu'ils rendent visible de manière saillante et temporellement resserrée, des processus de renormalisation, présents certes ailleurs, mais de manière beaucoup plus cachée et «dispersée temporellement». À ce titre, ils ne sont pas exemplaires de ce qui se passe en général, mais de ce qui peut se passer, voire de ce qu'il est probable qu'il se passe sans qu'on arrive toujours bien à le repérer.

\section{2.- Analyse d'une séquence d'alloconfrontation}

L'analyse qui suit porte sur un extrait de 10 minutes issu d'une séquence de travail sur la plateforme Néopass@ction. Le film visionné, Accueil par la présence physique, fait partie du thème 1 de la plateforme: L'entrée en classe et la mise au travail. Dans ce film, l'enseignante a recours à l'usage de modalités de communication non verbales, notamment le regard, qui lui permettent de faire autorité dans la classe. L'EEF a visionné en silence la plus grande partie du film, quand démarre l'extrait analysé.

La description que nous donnons de cet extrait n'est pas totalement neutre : nous ne donnons pas le verbatim tel quel. Nous tentons de rendre saillants les différents épisodes à l'œuvre dans l'élaboration par l'EEF et de l'activité visionnée et de sa propre activité. Par exemple : passage d'une description plus ou moins «neutre» de ce qui est vu à un point de vue plus interprétatif (thématisation par exemple des intentions ou des préoccupations de l'enseignante visionnée), passage d'une description à une appréciation de l'activité (par exemple, ce que fait l'enseignant marche bien), passage à des tentatives d'explication de ce qui se passe (par exemple, c'est parce que l'enseignant est calme que la classe est calme), changement d'objet (son activité propre à la place de l'activité visionnée), «surgissement » d'une nouvelle thématique, etc.

\section{1.- Description de l'extrait}

1. Après avoir arrêté le défilement du film, l'EEF initie son propos par une description d'événements suivie d'une appréciation de l'activité : ils sont très calmes ces élèves... elle a mis un peu de temps au début pour les placer, mais après ça a l'air de jouer assez. bien. ${ }^{2}$

2. Le CF lui demande si elle a observé comment elle s'y prenait. L'EEF produit une description d'événements, elle se met au milieu parmi eux, elle regarde l'élève à qui elle demande de faire quelque chose avec insistance jusqu'à ce qu'il fasse ce qu'elle lui a demandé.

3. Elle interprète l'activité de l'élève, le gamin il essaie quand même, puis compare l'activité de cet élève à celle de ses élèves à elle, moi aussi j'ai des élèves qui veulent s'asseoir là où ils ne doivent pas s'asseoir.

2 Les parties en italique sont des extraits du verbatim. 
4. Le CF essaie de la pousser vers une explication, qu'est-ce qui fait que d'après vous ça marche. Après avoir répondu qu'elle n'en sait rien, elle produit une suite d'explications : il y a un effet de personnalité, elle est très calme et posée, son travail a l'air structuré, elle est agréable, c'est peut-être une bonne journée, etc.

5. Cette suite d'explications concernant la situation filmée débouche sur une explication de sa propre activité : quand elle est calme et reposée, que son programme est bien établi, ça va beaucoup mieux se passer que quand elle est énervée et fatiguée.

6. L'EEF entre alors dans une comparaison de deux activités propres. Elle oppose les jours où elle laisse plus facilement tomber, où elle ne se focalise pas sur des choses (faire enlever les vestes par exemple) aux jours où elle s'acharne et se fixe là-dessus. Cette opposition « lâcher-prise $v s$ fixation » fait l'objet de plusieurs énoncés, dont la conclusion générale est que l'EEF n'est pas toujours au clair avec elle-même, est des fois prisonnière des règles qu'elle se donne, mais que quand elle laisse tomber certains côtés disciplinaires un petit peu superflus, ça va mieux. Cette attitude est plus en accord avec sa personnalité, elle estime qu'elle doit donc apprendre à être plus au clair.

7. Apparaît à ce moment, la thématique des normes institutionnelles exogènes : mais je le fais [entendre par là la fixation sur certains comportements des élèves] parce qu'on m'a dit qu'il fallait le faire.

8. Cette référence aux normes exogènes est illustrée par trois situations vécues par l'EEF :

9. Sa formatrice de terrain lui a une fois fait le reproche d'avoir laissé certains de ses élèves mâcher des chewing-gums. Elle-même n'avait rien remarqué et était d'ailleurs plutôt contente d'une leçon qui s'était bien passée.

10. Une autre fois, c'est une élève qui lui a fait remarquer qu'une autre élève avait un chewing-gum : du coup j'ai dit Leïla ton chewing-gum... mais moi j'avais même pas remarqué qu'elle avait un chewing-gum... et puis personnellement ça m'a pfff voilà... c'est une bonne élève...

11. Lors d'une visite du directeur de son établissement dans sa classe, celui-ci lui a fait part de certains comportements des élèves qui l'avaient profondément dérangé : moi je lui ai dit au directeur je lui ai dit ah bon, mais alors vous avez qu'est-ce qui vous a dérangé euh... parce que... peut-être moi j'ai pas remarqué...

12. L'EEF en conclut qu'elle doit devenir plus attentive pour les prochaines fois, mais d'un autre côté je me dis ben c'est comme ça... c'est comme ça... Elle répète qu'il faut qu'elle soit peut-être plus attentive effectivement à certains détails...

13. Le CF lui fait remarquer qu'en même temps elle semble dire que cela va mieux quand elle est moins focalisée sur les détails, ce qu'elle confirme d'un oui bien marqué.

14. L'EEF «contextualise » ensuite sa visée d'être plus attentive : c'est clair que maintenant je sais que quand les gens de l'école mes supérieurs viennent faut que je sois un peu plus attentive à certaines choses qui sont importantes pour eux...

15. Quand le CF explicite son propos comme une intention de donner une image, elle répond : oui ou peut-être qu'ils ont raison hein et que c'est moi qui ai tort... finalement je ne sais pas...

16. Elle revient ensuite sur la troisième situation en exprimant un conflit d'appréciations de son activité : mais en tous cas ça m'avait fait... ça m'avait frappé parce que moi ça m'était complètement passé...j'avais pas... c'était des travaux de groupe en plus donc... ça m'avait pas frappé que... pour moi c'était normal qu'il y ait plus de bruit et puis des choses comme ça...

17. L'EEF «conclut» cet extrait: donc pour dire que des fois euh... (montre l'écran) j'aimerais bien être comme elle... 


\section{3.- Synthèse du processus}

Le processus que nous avons décrit se structure en plusieurs phases.

Une première phase $(1,2,3)$ consiste en un mélange de descriptions d'événements, d'interprétation et d'appréciation de l'activité visionnée.

Une deuxième phase (4) est dévolue, suite à la demande du CF, à tenter d'expliquer l'activité visionnée. On peut dire des explications de l'EEF qu'elles sont «externalistes » au sens où les causes de ce qui se passe sont placées dans des «entités» externes au déploiement de l'activité elle-même, détachées de celle-ci et la précédant : ce sont les traits de caractère de l'enseignante, ou la «qualité » de la situation, qui sont les causes de ce qui se passe. Dans ce sens on peut dire que l'EEF échoue à exprimer quels sont les ressorts internes de l'activité visionnée.

Les explications «externalistes »données à l'activité visionnée par l'EEF ouvrent sur la thématisation de sa propre activité : elle donne à celle-ci des explications tout autant « externalistes » (5).

Cela l'amène dans une phase suivante (6) à confronter deux modes d'activité propres, celui où elle «lâche prise » et celui où elle reste «fixée» sur des règles. Cette confrontation aboutit à l'explicitation d'une contradiction entre deux règles soutenant son action. L'EEF exprime cette contradiction comme étant personnelle, endogène, et comme devant être dépassée : elle n'est pas claire et doit apprendre à l'être plus.

Dans la phase suivante (7), une nouvelle pièce est apportée à la discussion : en fait si elle reste fixée sur certaines règles, c'est parce qu'on lui a dit qu'il fallait le faire. Ce moment est à notre sens crucial dans la mesure où il introduit une sorte de rupture dans l'élaboration de son activité par l'EEF: elle reconnaît que celle-ci est contrainte par des normes institutionnelles qui ne sont pas de son fait. Bref, elle dépersonnalise la contradiction dans laquelle son activité est prise, en d'autres mots elle extrait la part exogène du conflit de normes du sein de l'endogène.

Toute la suite $(8,9,10,11,12,13)$ consiste à « tourner » autour de ce conflit de normes, par le recours à des anecdotes et l'expression de conflits d'appréciation : pour elle ça se passe bien, pour les «autres» non. Ce conflit de normes n'est pas clairement tranché : l'EEF ne sait pas qui d'elle ou « eux » a raison.

La dernière phase (14), qui montre une sorte de «retour» à l'activité visionnée peut être interprétée comme une sorte de projection sur l'enseignante visionnée à qui est prêtée la capacité à ne pas être prisonnière de la contradiction de l'EEF, projection qui aboutit au projet de dépassement de cette contradiction. Ce qui semble en dernier lieu laisser entendre que le conflit de normes a quand même été (certes non explicitement) tranché, qu'un processus de renormalisation a été mis en mouvement : l'exemple de l'enseignante visionnée a fait pencher la balance.

Il semblerait ainsi que c'est la difficulté même à saisir les ressorts internes de l'activité visionnée qui pousse l'EEF :

1. à énoncer des explications « externalistes »;

2. à tenter de valider ces explications en parlant de sa propre activité ;

3. à découvrir dans celle-ci des contradictions (moment d'explicitation);

— qu'elle thématise d'abord comme personnelles (normes endogènes);

— qu'elle élabore dans un second temps comme prenant leur source dans un conflit entre ses normes propres et les normes institutionnelles (extraction de l'exogène du corps de l'endogène);

4. à projeter sur l'enseignante visionnée une capacité à ne pas être prise dans ce genre de contradictions ; 
5. à «esquisser implicitement » un projet personnel - être plus claire, plus calme, lâcher prise - sur la base d'un processus de renormalisation.

\section{3.- Analyse d'une séquence d'autoconfrontation simple puis croisée}

L'analyse qui suit porte sur un extrait de 6 minutes issu d'une autoconfrontation d'une enseignante stagiaire d'allemand (classe d'élèves de 12-13 ans) puis d'un extrait de 2 minutes d'une autoconfrontation croisée entre deux enseignantes stagiaires d'allemand. La thématique que ces deux enseignantes ont convenu d'investiguer est la gestion des transitions (entre différentes activités individuelles, en groupe, collectives) dans la classe. Dans cet extrait, l'enseignante s'arrête sur un épisode durant lequel elle a mis les élèves au travail par deux pour répéter des dialogues en allemand, un épisode sur lequel la discussion reprend également lors de l'autoconfrontation croisée.

\section{1.- Description de l'extrait}

1. Le CF arrête le film pour demander quels sont les repères que l'EEF prend pour interrompre le travail en duo. L'EEF livre son vécu de la classe : là je doute beaucoup parce que c'est devenu assez bruyant je trouvais, moi. Je trouvais c'était trop bruyant.

2. L'EEF montre son insatisfaction par rapport à son activité : c'est assez problématique... Je ne suis pas du tout contente parce que je les interromps deux fois. Premièrement pour la consigne et maintenant encore. Mais c'était vraiment trop bruyant pour moi... Mais j'ai toujours un doute là-dessus. Est-ce que c'est trop bruyant ou est-ce que c'est moi ? Apparaît ici un questionnement sur ses normes concernant le niveau sonore acceptable dans la classe.

3. Dans le film visionné, l'EEF affiche une indication au rétroprojecteur indiquant le niveau sonore attendu lors de ce temps de travail. L'EEF décrit ses préoccupations à ce moment-là : je vois que c'est trop bruyant alors j'essaie avec ça parce que je ne veux pas trop crier. Ils sont tous dans leur activité alors je pense qu'en mettant une image...

4. Elle décrit également une autre façon d'agir qu'elle aurait pu adopter : Bon je peux aussi aller vers eux s'il y en a certains qui parlent trop fort. J'ai pas besoin... Mais ça devrait... Je peux pas laisser passer ça. Lorsque le CF lui demande si ce niveau sonore relève d'une sorte de norme un peu inconsciente qu'elle a sur ce que devrait être le niveau sonore dans une classe, l'EEF acquiesce : Je pense oui. Je pense c'est vraiment ça et c'est pas du tout pour la caméra car c'est vraiment souvent.

5. En continuant à visionner le film, l'EEF décrit et interprète l'activité des élèves : Je rappelle une règle, mais je les coupe aussi car je ne sais pas si ça se voit, mais ils se calment, je peux leur dire dans le calme, mais après ils osent plus parler les pauvres. Le CF propose de continuer le film, l'EEF décrit : Non ça ne se voit pas beaucoup [...], mais c'est dommage parce que c'est un dialogue de cinq lignes. Donc de le lire deux fois ça prend pratiquement pas de temps et là je les ai interrompus deux fois.

6. L'EEF amène une nouvelle explication: Je pense que j'avais quand même peur... Oui j'avais quand même peur qu'ils ne soient plus concentrés... Je sais pas c'était un mélange je pense... j'avais peur que je ne puisse plus les ramener après parce que c'était trop.

7. Lors de l'autoconfrontation croisée, l'EEF revient sur son vécu de ce moment qu'elle partage avec l'EEF 2 : C'est vrai que je regrette un peu parce que c'est un petit dialogue, ça ne prend pas longtemps et je les reprends deux fois. Je les reprends une fois pour la consigne et là je les reprends pour le bruit parce que j'avais l'impression... Je ne sais pas si ça se voit dans le film ou si c'est mon imagination, c'est mon gros problème. Et moi j'avais l'impression que vraiment c'était trop bruyant alors je les reprends encore une 
fois.

8. L'EEF 2 réagit : Non on n'a pas vraiment... J'ai l'impression que c'est une bénédiction moi (rires) et qu'ils sont tous dans leur dialogue en allemand. C'est peut-être simplement ça...

9. L'EEF revient alors sur sa sensibilité au niveau sonore : Mais c'est très très... Pour moi c'est très fort. C'est vraiment des moments où je commence à stresser de me dire... je pense que c'est des choses bêtes, de me dire si quelqu'un passe dans les couloirs et nous on fait un de ces brouhahas... Des fois j'entends à côté des portes et je vois comment font les autres parce que je veux savoir quel est le taux de volume...

10. L'EEF 2 propose de prendre comme critère plutôt le fait que les élèves parlent en allemand que de tenter de définir un niveau sonore «standard ». L'EEF interprète : Je pense que dans ces moments je suis... c'est-à-dire ridicule parce que là en plus dans le film vraiment on voit qu'ils travaillent bien, mais c'est vrai que c'est des moments où je suis très stressée... C'est l'impression que je dois quand même réagir maintenant alors je prends pas forcément le temps de voir [ce que font les élèves].

11. L'EEF 2 poursuit : En même temps, faut te dire que si tu leur demandes de parler... il y en a vingt-quatre qui interagissent ben c'est normal que le niveau sonore augmente et ça c'est dans tous les cours aussi.

12. L'EEF revient sur la peur qu'elle éprouve quand le niveau sonore monte : C'est peutêtre une peur que j'ai qui vient de l'année passée ou je sais pas C'est pas que j'ai peur qu'ils ne soient pas tous en train de travailler, j'ai peur que je vais les perdre, c'est toujours la même peur. J'ai peur qu'ils commencent à être vraiment bruyants et certains probablement j'ai vu qu'ils commencent à discuter et c'est cette peur-là... Je sais pas si à ce moment c'est vraiment justifié, mais...

13. L'EEF 2 abonde en son sens : Je crois que ça fait peur de laisser les élèves travailler par deux ou en groupe de manière autonome puis après de devoir les reprendre, c'est stressant. C'est d'ailleurs pour cela que certains ne le font pas, j'ai pas vu dans tous les cours d'allemand du travail entre partenaires.

14. L'EEF conclut: Mais je pense que c'est vraiment une histoire de... maintenant si j'y réfléchis, une histoire de regard extérieur. J'ai peur que quelqu'un de l'extérieur passe... et c'est ça, la peur... comme quand je suis en ville avec ma fille et je dois l'engueuler et j'ai peur des regards c'est la même... je pense que c'est le même stress...

\section{2.- Synthèse du processus}

Nous repérons plusieurs phases dans ces extraits. Une première phase de l'autoconfrontation simple (1 à 5) consiste en l'expression du vécu, des émotions et des préoccupations de l'EEF qu'elle énonce à travers des descriptions, des interprétations et des appréciations de son activité en classe. Apparaît d'abord un questionnement au niveau des règles implicites que l'EEF se donne et qui mettent en tension les règles endogènes et exogènes concernant le niveau sonore acceptable dans la classe. On voit ici émerger un conflit qui l'habite entre arrêter les élèves qui travaillent en duo versus accepter un niveau sonore qu'elle juge trop élevé, conflit qui oppose deux «règles » exogènes et implicites de métier: «il ne faut pas interrompre des élèves qui travaillent » et « les élèves ne peuvent pas travailler si le niveau sonore est trop élevé ».

Dans une deuxième phase de l'autoconfrontation (6), l'EEF associe ce conflit à la peur qu'elle éprouve de ne pas être capable de reprendre la classe en main si les élèves sont trop excités.

Au moment de l'autoconfrontation croisée, l'EEF revient dans une première phase $(7,8)$ sur le conflit qui l'habite qu'elle expose à l'EEF 2 en décrivant ce qu'elle voit dans le film ainsi que ses interprétations et appréciations relatives au niveau sonore. L'EEF 2 lui propose une interprétation différente. 
Suit une phase (9 à 11) durant laquelle l'EEF va creuser la question de l'effet du niveau sonore sur ses émotions (stress, peur) et identifier une première peur qui l'habite, celle du regard extérieur que pourrait poser sur son activité une personne extérieure à la classe. Elle réalise que cette peur prend tellement de place chez elle qu'elle l'empêche de prendre en compte et d'apprécier l'activité des élèves.

Lors de la phase suivante $(12,13)$, l'EEF va mettre en parallèle deux évènements : la peur qu'elle éprouve dans cette séquence avec celle qu'elle a éprouvée l'année précédente, à savoir perdre le contrôle sur la classe et sur le travail des élèves. L'EEF 2 reconnaît cette peur comme légitime.

Enfin, lors de la dernière phase (14), l'EEF met cette fois-ci en parallèle les intentions qu'elle a dans la classe et dans d'autres situations, à savoir la gêne face au regard qu'une personne extérieure pourrait porter sur son attitude d'enseignante/de mère.

On voit que durant ce double extrait (autoconfrontation simple puis croisée), l'EEF part de la gêne qu'elle éprouve par rapport à un niveau sonore qu'elle juge élevé qui 1) la plonge dans un conflit entre laisser dialoguer en acceptant le niveau sonore ou interrompre les élèves au risque de casser la dynamique d'expression en allemand 2) «obscurcit» sa capacité à apprécier l'activité de ses élèves et la pertinence de son dispositif didactique. À travers les interactions avec le CF puis avec l'EEF 2, elle va essayer d'expliciter ce sentiment de gêne, en identifiant une double peur/stress qui conditionne son comportement : peur de perdre le contrôle sur les élèves et peur face aux attentes extérieures (peur du regard sur sa légitimité d'enseignante, de mère). L'enquête sur son activité lui a donc dans un premier temps, permis d'expliciter un conflit de normes d'abord présenté comme endogène. Dans un second temps, elle a pu extraire la part exogène du conflit de normes du sein de l'endogène.

\section{4.- Discussion}

On peut relever des similitudes dans les deux cas analysés. Dans un premier temps, les règles implicites organisant l'activité font l'objet d'une explicitation langagière. Cette explicitation permet de rendre visible un conflit de normes données comme endogènes : les contradictions sont exprimées comme étant des contradictions personnelles. Mais dans un second temps, on assiste à une "dé-personnalisation» du conflit : celui-ci est réélaboré comme un conflit entre normes endogènes et normes exogènes (les attentes implicites ou explicites du milieu). Il semble donc qu'un processus de renormalisation s'initie à l'aide d'un double mouvement : passage de l'implicite à l'explicite, identification et extraction (ou objectivation) de l'exogène du sein de l'endogène.

Ceci nous amène au constat suivant : si renormaliser consiste en suivant Schwartz (Schwartz, 2007 ; Schwartz \& Durrive, 2009) à réinterpréter les normes émises par le milieu en vue de configurer ce milieu comme étant le sien (à rendre ce milieu personnellement viable), encore faut-il être capable d'identifier ces normes comme étant celles du milieu et non comme les siennes. Cette confusion entre normes endogènes et exogènes est une des difficultés que rencontrent les enseignants débutants et l'on voit que les dispositifs d'alloconfrontation et d'autoconfrontation peuvent permettre de la travailler, voire de la dépasser. Ces dispositifs sont donc à inscrire au sein d'environnements de formation visant à « redonner la main » aux enseignants en leur permettant de mieux comprendre ce qui est significatif pour eux, ce qui va perturber leur activité et déclencher une transformation de celle-ci : c'est dans ce sens que nous reprenons la position de Dewey (1990) qui considère que les objectifs de formation doivent viser en premier lieu à libérer l'activité.

Par ailleurs, si l'on s'intéresse aux origines du processus de renormalisation relevé dans nos deux cas, on voit que celles-ci sont distinctes. Il est suscité :

— dans le premier cas par une norme différente provenant de l'activité d'autrui (alloconfrontation); 
— dans le second cas par le visionnement de sa propre activité (autoconfrontation) et

l'apport de normes différentes par un pair durant l'autoconfrontation croisée.

Dans le premier cas, il n'y a pas d'objet d'enquête prédéfini avant le visionnement du film. Un objet émerge dans la confrontation à l'activité d'autrui. Celle-ci, par contraste, opère comme un «révélateur»: les normes de l'activité propre de l'EEF, normes que celle-ci estime "pas claires », sont opposées à celles, jugées «claires» de l'enseignante visionnée. Mais l'EEF ne parvient pas à entrer dans l'analyse des ressorts internes de l'activité visionnée, les explications qu'elle en donne restent externalistes. Par là, elle n'arrive pas à «aller plus loin » que cette expression d'un conflit de normes : sa formulation d'un projet personnel, «être plus claire», reste vague et ne se concrétise pas dans l'élaboration de possibilités de transformation de son activité. Deux pistes permettant de prolonger le processus de renormalisation initié et de «libérer l'activité » de l'EEF pourraient être envisagées de manière complémentaire.

La première consisterait à guider l'EEF dans une description fine de l'activité visionnée, afin qu'elle puisse dépasser le simple constat que «ça marche». Il s'agirait de la pousser à passer d'un registre évaluatif à un registre descriptif, afin de lui permettre de formuler, puis de valider ou invalider, des hypothèses concernant les organisateurs de l'activité de l'enseignante et des élèves (Lussi Borer \& Muller, 2014). Elle pourrait ensuite tester ces hypothèses sur sa propre activité.

Une deuxième piste consisterait à proposer à l'EEF de mener une enquête sur la mise en œuvre des normes relatives au bruit, au chewing-gum, etc., dans son activité en classe via un enregistrement vidéo. Suivrait une autoconfrontation qui, sur la base du repérage de ce qu'elle vit à ce propos, permettrait d'envisager avec elle des bifurcations possibles de son activité.

Dans le second cas, un objet d'enquête, «la gestion des transitions», est défini préalablement aux visionnements du film en autoconfrontations simple et croisée. À partir de l'analyse de l'activité de gestion de la transition lors de l'autoconfrontation simple, l'EEF élabore un conflit de normes à propos du niveau sonore en classe. Elle initie alors une enquête sur l'activité des élèves pour valider/invalider la pertinence de son interruption de cette activité durant le dialogue en allemand. Son état de doute persiste toutefois et elle choisit de revenir sur cette situation en autoconfrontation croisée pour avoir l'avis de sa collègue. L'EEF 2 convoque des dimensions internes à l'activité des élèves pour nourrir l'enquête : les élèves parlent allemand, sont dans le dialogue. L'EEF admet alors que les élèves sont au travail. Ce constat lui permet de revenir sur son conflit de normes et de prendre conscience que c'est la peur d'un regard extérieur qui suscite celui-ci. En termes de formation, on voit que l'EEF reste sur cette explication externaliste de son activité et qu'il y aurait là, des pistes de transformation portant sur son activité en classe à co-construire et à tester, pour poursuivre l'enquête et développer le processus de renormalisation initié.

\section{Conclusion : quelles pistes pour la formation ?}

La comparaison de ces deux cas emblématiques semble montrer que nous sommes face à un processus similaire même s'il est généré par des dispositifs différents. Nous faisons l'hypothèse suivante: l'alloconfrontation opèrerait comme une sorte d'autoconfrontation fictionnelle - elle permet de regarder l'activité d'autrui comme si c'était la sienne - et l'autoconfrontation opérerait comme une sorte d'alloconfrontation fictionnelle : elle permet de regarder sa propre activité comme si c'était celle d'autrui. Et dans les deux cas, ce qui compterait, quelle que soit sa direction, c'est ce mouvement d'altération de sa propre activité, altération porteuse du double mouvement d'explicitation de l'implicite et d'extraction de l'exogène du corps de l'endogène.

Il nous semble pertinent, dans une visée de développement d'environnements de formation, de mieux s'intéresser à ce «faire comme si ». En effet, cette immersion fictionnelle, cette 
expérience mimétique (Durand, Goudeaux, Horcik, Salini, Danielian, \& Frobert, 2013), offre des registres d'expériences qui se situent sur un rapport d'analogie globale et non d'homologie. La capacité à se projeter dans des situations proches, mais pas identiques, qui ont un «air de famille» avec celles déjà rencontrées (ce n'est pas ce que je fais, mais pas pas ce que je fais), semble permettre de «dégager» des dimensions «en germe» dans l'activité, qu'une trop grande proximité - tout comme un trop fort éloignement - ne permet pas d'appréhender. Comme le montre Zaccaï-Reyners, cette immersion fictionnelle suscite « des états de conscience propices à l'exploration des possibles sur un mode mineur » (2005, p. 17), qui autorisent la personne en formation à se projeter dans d'autres rôles possibles sans pour autant mettre trop en danger son identité professionnelle. Il reste toutefois que cette projection ne dit rien sur son opérationnalisation dans une activité ultérieure : le passage du « je pourrais faire autrement » à «j'essaye de faire autrement » représente ainsi un enjeu de formation qu'il s'agit d'investiguer.

En effet, nous observons dans les deux cas analysés que les conflits de normes constituent de bons objets d'enquête potentiels, représentatifs de dilemmes propres à la culture du métier d'enseignant, mais qu'ils ne peuvent évoluer sans être ramenés aux dimensions opérationnelles de l'activité qui fonctionnent à la fois 1) comme analyseurs de l'expression du conflit de norme dans la situation analysée, 2) comme pistes de transformation (notamment à travers l'activité d'autrui), 3) comme lieux d'exercice des transformations projetées, 4) comme lieux de validation de la pertinence de l'activité transformée. Ainsi, prolonger ce type d'expérimentation avec des boucles d'enquêtes collaboratives allant jusqu'à la mise en œuvre des pistes de transformation envisagées serait un objectif pour développer la formation (Ria \& Lussi Borer, 2013).

L'enjeu principal est donc de concevoir une ingénierie de formation à même d'outiller les EEF en responsabilité pour leur permettre de dépasser des explications externalistes et de revenir aux dimensions opérationnelles de l'activité. Parmi les dimensions génériques à inclure dans cette ingénierie de formation, les suivantes nous apparaissent cruciales.

1) Faire prendre conscience du fait que l'on porte toujours «spontanément» un regard normatif sur l'activité et susciter la suspension momentanée de ce regard normatif en faveur d'un regard descriptif plus «neutre », puis d'un regard interprétatif permettant d'analyser finement les ressorts de l'activité développée par l'enseignant visionné et de comprendre son vécu (intentions, émotions et perceptions) (Lussi Borer \& Muller, 2014).

2) Proposer la co-construction collective d'un ou de plusieurs objet(s) d'enquête sur une ou des dimension(s) identifiée(s) de l'activité. Proposer un étayage conceptuel sur lequel ancrer l'enquête et des principes éthiques afin qu'elle puisse se dérouler dans un climat de confiance et de bienveillance.

3) Fournir les outils technologiques et méthodologiques permettant de mener l'enquête (notamment en ce qui concerne la réalisation d'entretiens d'autoconfrontations simple, croisée et collective).

4) Mettre en place des petits groupes d'EEF qui mènent leur enquête collaborative de manière autonome, le formateur les accompagnant en fonction de leurs besoins.

Une telle ingénierie de formation rend possible des processus personnels d'explicitation-désendogénéisation, produits au sein de collectifs d'enseignants en formation. Ces collectifs fonctionnent comme ressources pour accompagner, enrichir, voire valider les projets de transformation de l'activité formulés par les individus. Nous faisons l'hypothèse qu'in fine, ces processus de formation participent aussi à la transformation de la culture du métier, du genre professionnel, etc., comme au renouvellement des savoirs professionnels formalisés et partagés. 


\section{BIBLIOGRAPHIE}

Clot, Y., \& Faïta, D. (2000). Genre et styles en analyse du travail. Concepts et méthodes. Travailler, 4, 7-42.

Clot, Y., Faïta, D., Fernandez, G., \& Scheller, L. (2000). Entretiens en autoconfrontation croisée : une méthode en clinique de l'activité. Pistes, 2, 1-7.

Cometti, J.-P. (2011). Qu'est-ce qu'une règle ? Paris: Vrin.

Dewey, J. (1990). Démocratie et éducation. Paris: Armand Colin.

Durand, M. (2008). Un programme de recherche technologique en formation des adultes. Éducation et Didactique, 2(2), 69-93.

Durand, M., Goudeaux, A., Horcik, Z., Salini, D., Danielian, J., \& Frobert, L. (2013). Expérience, mimèsis et apprentissage. In L. Albarello, J.-M. Barbier, E. Bourgeois \& M. Durand (Eds.), Expérience, activité, apprentissage (pp. 39-64). Paris: PUF.

Garetta, G. (2002). Une régularité sans répétition ? L'habitude comme schème dynamique. In C. Chauviré \& A. Ogien (Eds.) La régularité. Habitude, disposition et savoir-faire dans l'explication de l'action (pp. 137-160). Paris: Editions de l'EHESS.

Lahire, B. (1998). L'homme pluriel. Les ressorts de l'action. Paris: Nathan.

Leblanc, S. (à paraître). Des dispositions concurrentes pour mener un entretien post-leçon : Étude des effets d'un contexte d'entretien « innovant». In I. Plazaola Giger \& A. Muller (Eds.), Dispositions, travail et Formation. Toulouse: Octarès.

Lussi, V., \& Muller, A. (2014). Quel apport/usage du « voir» pour le «faire » en formation des enseignants du secondaire. In M. Altet, R. Etienne, L. Paquay, \& P. Perrenoud (Eds.), Comment la formation des enseignants prend-elle en compte la réalité du travail enseignant et les prescriptions dont il fait l'objet dans le milieu scolaire ? Bruxelles: De Boeck.

Lussi Borer, V., \& Muller, A. (à paraître a). Connaître l'activité des enseignants en formation sur la plateforme Néopass@ction.Recherche et Formation.

Lussi Borer, V., \& Muller, A. (à paraître b). Analyse de l'activité, environnements de formation et développement professionnel. Différences intra et inter-activités : le possible, le réel et le virtuel. Recherche et profession.

Méard, J., \& Bruno, F. (2009). Les règles du métier dans la formation des enseignants débutants. Études de cas dans le primaire et le secondaire. Toulouse: Octarès.

Muller, A. (2014). Conclusion : retour sur la conception des dispositions développée dans cet ouvrage. In In I. Plazaola Giger \& A. Muller (Eds.), Dispositions, travail et Formation (pp. 189-195). Toulouse: Octarès.

Ria, L., \& Lussi Borer, V. (2013). «Laboratoire d'enseignants encore apprenants » au sein d'un établissement scolaire : enjeux, méthodes et effets sur la formation des enseignants. Actes du Congrès AREF 2013 "Actualité de la Recherche en Education et Formation », Université de Montpellier : http://www .aref2013.univ-montp2.fr/cod6/?q=content/\%C2\%AB-laboratoire$\mathrm{d} \% \mathrm{E} 2 \% 80 \% 99$ enseignants-encore-apprenants-\%C2\%BB-au-sein-d\%E2\%80\%99un$\% \mathrm{C} 3 \%$ A9tablissement-scolaire-enjeux-0

Schwartz, Y. (2007). Un bref aperçu de l'histoire du concept culturel d'activité. Activités, 4(2), $122-$ 133.

Schwartz, Y., \& Durrive, L. (Eds.) (2009). L'activité en dialogues. Entretiens sur l'activité humaine (II). Toulouse: Octarès.

Theureau, J. (2010). Les entretiens d'autoconfrontation et de remise en situation par les traces matérielles et le programme de recherche « cours d'action ». Revue d'anthropologie des connaissances, 4(2), 287-322.

Venner, B., \& Schwartz, Y. (2009). Débats de normes, «monde des valeurs » et engagement transformateur. In Y. Schwartz \& L. Durrive (Eds.), L'activité en dialogues. Entretiens sur l'activité humaine (II) (pp. 35-99). Toulouse: Octarès.

Winch, P. (2009). L'idée d'une science sociale et sa relation à la philosophie. Paris: Gallimard.

Zaccaï-Reyners, N. (2005). Fiction et typification. Contribution à une approche théorique de la transmission de l'expérience. Methodos, 5. Retrieved from https://methodos.revues.org/378

Zask, J. (2004). L'enquête sociale comme inter-objectivation. In B. Karsenti \& L. Quéré (Eds.), La croyance et l'enquête. Aux sources du pragmatisme (pp. 141-163). Bruxelles: De Boeck. 


\section{RÉSUMÉ}

Cet article analyse le potentiel de renormalisation contenu dans les dispositifs de formation «orientés activité » utilisant le visionnement de films montrant des enseignants au travail. Par renormalisation, nous entendons le travail permanent de renouvellement des règles d'élaboration de l'expérience et des règles à suivre dans l'action. Nous soumettons à analyse une séquence d'alloconfrontation et une séquence d'autoconfrontation simple suivie d'une autoconfrontation croisée produites dans le cadre d'une recherche menée à l'Université de Genève avec des enseignants secondaires en formation. Les processus de renormalisation identifiés font l'objet d'une description fine, suivant pas à pas le développement de l'interaction des enseignants avec les films visionnés. L'analyse montre que ces processus de renormalisation reposent sur un double mouvement d'explicitation de règles implicites et d'élaboration de conflits entre les règles personnelles et les règles du milieu (institution et attentes des pairs). Nous discutons ensuite le potentiel de ces processus pour la formation et proposons une ingénierie à même de les favoriser.

\section{MOTS CLEFS}

enseignement, vidéo-formation, activité, renormalisation

\section{REFERENCEMENT}

Lussi Borer, V., \& Muller, A. (2014). Exploiter le potentiel des processus de renormalisation en formation à l'enseignement. Activités, 11(2), 129-142. http://www .activites.org/v11n2/v11n2.pdf

Article reçu le 31 janvier 2014, accepté pour publication le 5 juin 2014 